Prof. Dr. med. C. Bayerl im Gespräch mit

Herrn Prof. M. Schaller:

\section{Moderne mykologische Diagnostik}

\section{Bayerl: Was ist Ihrer Ansicht nach die Basis mykologischer} Diagnostik?

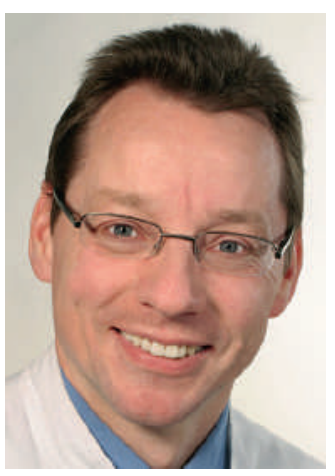

M. Schaller: Die Basis der mykologischen Diagnostik ist immer noch das gute alte Nativpräparat und die kulturelle Diagnostik. Der Vorteil des Nativmaterials ist ganz klar: Es ist billig, einfach und liefert schnelle Ergebnisse. Sie können damit zeigen, ob es sich um eine Infektion der Haut handelt oder nicht. Der kulturelle Nachweis hat natürlich ein gewisses Zeitfenster von 4-6 Wochen, welches der Pilz braucht, um auf diesen Nährböden zu wachsen. Das ist wichtig für die genaue Bestimmung der Spezies. Diese Einordnung ist zwar therapeutisch vielleicht nicht so wichtig, hilft aber Infektionsketten zu finden. Wenn Sie z. B. eine zoophile Dermatophyteninfektion nachweisen, dann muss das Tier mitbehandelt werden, und deshalb ist es wichtig, eine genaue Speziesbestimmung zu machen.

C. Bayerl: Welche Weiterentwicklungen hat es bei der mykologischen Diagnostik gegeben?

M. Schaller: Auch hier haben die molekularbiologischen Methoden Einzug gehalten. Es ist heute einfach möglich, mit einer PCRUntersuchung Sofortdiagnostik zu betreiben. Innerhalb von 24 Stunden kann eine Spezies-Diagnostik vorliegen. Dies bietet sich auch an, wenn nur wenig Material zur Verfügung steht. Die Empfindlichkeit dieser PCR-Diagnostik ist deutlich höher als die Empfindlichkeit des Nativpräparats und der Kultur. Dazu ist aktuell eine Studie an der Hautklinik Tübingen, München und in einer Hautarzt-Praxis in Ulm durchgeführt worden.

C. Bayerl: Wie sieht ein Modell aus, um Candida-Infektionen zu untersuchen?

M. Schaller: Wir haben viel Erfahrung in der Etablierung von Candida-Infektionsmodellen. Hierbei handelt es sich um sogenannte in-vitro-rekonstituierte humane Haut oder humane Schleimhaut. Die Methodik basiert auf der Verwendung einer Zelllinie, die auf einer Polykarbonat-Unterlage aufgebracht wird und ein mehrschichtiges Abbild der menschlichen Haut oder Schleimhaut bildet. Eigentlich kommen diese Modelle aus der pharmakologischen Testung und werden in großem Umfang von bestimmten Firmen zur pharmakologischen Irritationsanalyse eingesetzt. Man kann aber diese Modelle sehr gut einsetzen, um Infektionen mit Candida albicans nachzuahmen. Der weitere Vorteil ist, dass in diesen Modellen dann auch wie in vivo bestimmte Immunzellen miteingebracht werden können und so die Interaktion zwischen den Keratinozyten, dem Erreger und den Immunzellen untersucht werden kann.
C. Bayerl: Gibt es immunologische Unterschiede zwischen Candia-Infektionen der Schleimhaut und Candida-Infektionen der Haut?

M. Schaller: Es gibt sehr gute Daten für immunologische Unterschiede zwischen der Schleimhaut oral und der Schleimhaut vaginal. Dort spielen deutlich andere immunologische Faktoren eine Rolle. Es zeigt sich schon alleine deshalb, dass Patienten mit HIV-Infektion eine hohe Rate an oralen Infektionen haben, aber eine niedrige Rate an vaginalen Infektionen und die angeborene Immunität wahrscheinlich im Vaginalbereich eine viel höhere Bedeutung hat. An der Haut kommen Candidosen eigentlich in erster Linie im intertriginösen Bereich vor, da hier eine erhebliche Barriereschädigung auftritt und diese Barriereschädigung ermöglicht es dem Erreger, in die Haut einzudringen. Wird diese Barriereschädigung entsprechend behandelt, ist eine kutane Infektion mit Candida eigentlich nur bei starker Immunsuppression oder bei der chronischen kutanen Candidose nachweisbar.

C. Bayerl: Inwieweit unterscheiden Sie invasive und nicht-invasive Candida-albicans-Stämme?

M. Schaller: Es ist wirklich so, es gibt nicht den C. albicansStamm, der immer gleichförmig virulent ist, sondern es gibt wirklich Stämme, die man vom Patienten isoliert mit deutlichen Virulenzunterschieden. Der wichtigste Faktor ist wahrscheinlich die Hyphenbildung. Wenn ein Stamm zur Hyphenbildung aus irgendwelchen Gründen nicht mehr fähig ist, und das weiß man durch Knock-out-Mutanten, dann ist eine Infektion kaum mehr möglich. Darüber hinaus gibt es aber auch Unterschiede bei anderen Virulenzfaktoren, z.B. der sekretorischen Aspartatproteinase. Es konnte gezeigt werden, dass es Stämme gibt, die viele dieser Proteinasen bilden und damit virulenter sind als Stämme, die wenig Proteinasen produzieren.

\section{Bayerl: Welche klinischen Konsequenzen folgen aus diesen} neuen Daten?

M. Schaller: Ich denke, dass die Arzneimittelentwicklung in eine andere Richtung gehen sollte. Es gibt einige sehr gute Antimykotika, aber die Resistenzentwicklung hat sie immer wieder eingeholt und die Konsequenz wäre eigentlich, dass man nicht nur mit den klassischen Antimykotika das Wachstum behandelt, sondern dass man auch versucht, Virulenzfaktoren zu hemmen und damit eben in einem zweifachen Ansatz auf den Erreger einwirkt, um eine bessere Wirkung zu erzielen.

C. Bayerl: Ich danke Ihnen für das Interview.

Prof. Dr. med. Martin Schaller

Universitäts-Hautklinik

Klinikum der Universität Tübingen

Liebermeisterstraße 25

72076 Tübingen

Martin.Schaller@med.uni-tuebingen.de 